The Astrophysical Journax, 208:L77-L81, 1976 September 1

(C) 1976. The American Astronomical Society. All rights reserved. Printed in U.S.A.

\title{
COMMENTS ON GRAVITATIONAL RADIATION DAMPING AND ENERGY LOSS IN BINARY SYSTEMS
}

\author{
JÜRgEN EHLERS AND ARNOLD ROSENBLUM \\ Max-Planck-Institut für Physik und Astrophysik, Munich, Germany \\ JOSHUA N. GOLDBERG \\ Department of Physics, Syracuse University \\ AND \\ Peter Havas \\ Department of Physics, Temple University \\ Received 1976 A pril 12; revised 1976 June 21
}

\begin{abstract}
It is argued that a formula for the energy loss due to gravitational radiation of bound systems such as binaries has not yet been derived either exactly or by means of a consistent approximation method within general relativity, a view which contradicts some widely accepted claims in the literature. The main approaches used to obtain such a formula are critically reviewed, and it is pointed out that the derivations presented so far either contain inconsistencies or are incomplete.
\end{abstract}

Subject headings: gravitation - relativity - stars: binaries

The determination of radiation reaction forces is a fundamental problem of any field theory of gravitation. More specifically, the discovery of the binary pulsar PSR $1913+16$ and systems such as Ariel 1118-61 has led to new interest in the energy loss and the associated damping of binary systems due to gravitational radiation (Paczynski 1967; Faulkner 1971; Pringle and Webbink 1975; Wagoner 1975; Chau and Lauterborn ${ }^{1}$ 1976). The purpose of this Letter is to point out that $a$ formula for the energy loss of such a system has still not been derived consistently within general relativity, contrary assertions in the literature notwithstanding. We hope that our comments will stimulate research to settle this basic question conclusively. In our opinion the statement in italics deserves to be emphasized since there is some danger of misinterpretation of observational data if certain familiar and "plausible" equations in the literature concerning these problems (the first of which was obtained by Einstein 1918 on the basis of the linearized theory) relating the loss of energy to the square of the third derivative of the quadrupole moment are considered as firmly established consequences of general relativity.

The idea of energy loss due to radiation, and a corresponding radiation reaction force in the equation of motion of a particle, arose in the theory of the electromagnetic field (Lorentz 1909), and it can be expected that any field theory of gravitation would similarly lead both to a loss of energy and to radiation reaction forces. Indeed, all plausibility arguments (for a particularly clear exposition see Kalckar and Ulfbeck 1974) attempt to exploit such an analogy. But we are

\footnotetext{
${ }^{1}$ This unpublished study includes an investigation of the (rather drastic) influence on the main sequence time scale of binaries of orbital angular momentum losses which might be due to gravitational radiation.
}

concerned here not with a field theory of gravitation in the abstract, but with a particular one: Einstein's general theory of relativity. While in some respects Einstein's theory indeed has the same structure and properties as Maxwell's electromagnetic theory, in some other respects it is fundamentally different (for a simple discussion see Havas 1973). Our skepticism concerning the usual familiar equations is not due to any disagreement concerning what is plausible, but arises from the technical question whether these equations have been established as a consequence of Einstein's theory with acceptable rigor, or whether their derivation has been based on assumptions some of which are mathematically incompatible with this theory.

Little is known rigorously about gravitational radiation from localized matter distributions. On the assumption of asymptotically flat spacetime in the vicinity of null infinity it has been shown (Sachs 1962; Newman and Unti 1962) that (except in certain special cases) null rays coming from an isolated matter distribution shear as they approach null infinity as seen in an asymptotically Minkowskian frame. The square of the rate of change of shear determines the rate of radiation of gravitational energy. What is missing is a connection between the shear and the observed motion of stellar systems, in particular their quadrupole moments, and thus it is not possible to translate the rigorously known result involving the shear into a numerically testable statement.

In order to obtain such a testable statement, it has been necessary to resort to approximation methods which are based on an iteration or expansion of the deviation of the physical metric tensor from the Minkowski flat space metric. These attempts have not yet arrived at a consistent solution. Here we shall outline 
the general approach to the problem of motion and briefly criticize some of the more prominent calculations.

The problem is to determine approximately spacetime models $(M, g, T)$ which fulfill the following conditions:

I) They satisfy Einstein's equation

$$
G=8 \pi T,
$$

and thus also its consequence

$$
\nabla \cdot T=0 .
$$

II) They correspond to a physically reasonable model of the sources (bodies). This can be achieved either by specifying the functional dependence of the stressenergy-momentum tensor $T$ on some matter variables $m_{A}$ and the metric $g$,

$$
T=T\left(m_{A}, g\right)
$$

as illustrated by two examples in the Appendix, or by requiring that in some world tube representing the region outside, but close to the body in question, the metric has asymptotically a specific form (e.g., Schwarzschild or Kerr) characteristic of a single body. ${ }^{2}$

III) They satisfy a condition for absence of incoming radiation. The precise form of the boundary condition (III) is not known and can perhaps be formulated only once a satisfactory solution of the whole problem has been obtained. Some such condition, however, is certainly needed to make the problem mathematically well defined and to express the physical assumption that the system has indeed been isolated except during some unknown prehistory. It is not known whether nonstationary spacetimes exist which possess a past null infinity $\mathfrak{G}^{-}$(in the sense of Penrose 1964), and which of them, if any, are free of incident radiation; in model theories Leipold (1976) has shown that it depends on the early motion of the sources (for $t \rightarrow-\infty$ ) whether retarded fields do or do not contain incident radiation. In view of these facts and the presently accepted view that the existence of $\mathscr{g}$ is an essential part of the definition of an isolated system, it appears to be premature to claim that gravitational radiation from isolated material systems is theoretically well understood, even in principle.

Equation (2) determines the law of motion of the bodies in terms of the (unspecified) metric $g$. To obtain the metric $g$ and the equations of motion, i.e., the laws of motion with the $g$ expressed in terms of the matter variables, one imposes a coordinate condition ${ }^{3}$

$$
C(g)=0
$$

such that equation (1) can be replaced by a relaxed field equation

$$
\hat{G}(g)=T\left(m_{A}, g\right)
$$

${ }^{2}$ The basic ideas of this method to describe bodies were already clearly formulated by Weyl (1923) and have recently been elaborated and extended by means of matched asymptotic expansions (D'Eath, 1975a, b).

${ }^{3}$ See, however, the remarks in the paragraph following eq. (7a). which no longer implies equation (2), so that equation (5) can be solved for arbitrary values of the $m_{A}$ 's. The restrictions on the motion follow by subjecting the solutions of equation (5) to the coordinate condition (4). Since the pair (4), (5) of equation is equivalent to equation (1), any solution of equations (4), (5) will satisfy equation (2) also. ${ }^{4}$ Moreover, equation (4) is chosen such that equation (5) is a simpler differential equation than equation (1) and can be solved, hopefully, by successive approximations.

The computational strategy is $(a)$ to solve equation (5) iteratively, by computing a sequence ${ }^{1} g,{ }^{2} g, \ldots$, where each approximate potential is a functional

$$
{ }^{N} g\left[m_{A}\right]
$$

of the matter variables obeying the boundary condition (III), and then (b) to impose on ${ }^{N} g\left[m_{A}\right]$ the condition (4),

$$
C\left({ }^{N} g\left[m_{A}\right]\right)=0 .
$$

It is essential that equation (7a) be imposed after the iteration has been performed to the desired order; requiring (7a) at each step would lead to mutually contradictory equations of motion on the matter variables.

A different iteration method not requiring any coordinate condition has been formulated by Havas and Goldberg (1962). In their procedure the $(N-1)$ th approximate equation of motion is the integrability condition for the $N$ th order approximate field equation. Instead of replacing equation (1) by a relaxed equation (5), these authors propose to solve successively linearized, but untruncated, approximations of equation (1). After each iteration step the solution ${ }^{N} g\left[m_{A}\right]$ is then relaxed by keeping the form of these functionals, but discarding the restrictions imposed on the $m_{A}$ 's by the lower-order equations of motion. The role of equation (7a) is then played by the equivalent equation

$$
{ }^{N-1} \nabla \cdot T\left(m_{A},{ }^{N-1} g\left[m_{A}\right]\right)=0 .
$$

However, so far no method of solving their $N$ th-order field equation without coordinate conditions has been found, so that for explicit calculations their method reduces to that described above.

According to either iteration scheme, to $N$ th order the evolution of an isolated system is described by an " $N$ th order metric" $N g$ satisfying the $(N-1)$ th order equation of motion (7a) or (7b). If these equations of motion can be cast into a Newtonian or special-relativistic form, radiation reaction forces can be identified by means of their time-reflection asymmetry. The radiation loss can be described either through a suitably defined function of the metric near future null infinity $\mathrm{g}^{+}$, interpreted as the flux of gravitational radiation

${ }^{4}$ Mathematically, this situation resembles that prevailing in special-relativistic electrodynamics. Maxwell's equations, whether expressed in terms of fields or of potentials, imply the equation of continuity; however, before solving the equations for the potentials they are simplified through introduction of a gauge condition. Then these equations no longer imply the equation of continuity, and the solutions must be restricted by means of the gauge condition. 
energy, or through the time rate of change of a suitably defined function of the source variables $m_{A}$, interpreted as the total energy of a bound system. The radiation condition (III) will ensure that the motion of the system is time-asymmetric, just as in mechanical and electromagnetic cases.

Thus it is an approximate metric together with matter variables satisfying (7a) or (7b) which governs the time development of a system. An approximate solution (6) of equation (5) which does not also satisfy the $(N-1)$ th equation of motion is not an approximate solution of Einstein's equation (1). Unfortunately, this fact was not properly taken into account in the two best known derivations of a formula for gravitational radiation loss of a system, Einstein's 1918 original derivation on the basis of the linear approximation ${ }^{5}$ and the substantially identical one given by Landau and Lifshitz (1951), who overlooked the restrictions on the motion implied by the field equations. (Similar derivations, also overlooking these restrictions, are given in many recent articles as well as textbooks such as Weinberg 1972 or Ohanian 1975.) It is also the same point at which some popular plausibility arguments go wrong; e.g., Kalckar and Ulfbeck (1974) argue the existence of a radiation reaction force in a two-body system by considering the work done by an external agent in overcoming such a force under various conditions including keeping one body fixed. However, Einstein's equations do not allow such a freedom; they allow only one particular motion under given initial conditions. Violating the equations of motion is analogous to considering solutions of Maxwell's equations which violate the equation of continuity.

In some subsequent derivations, the field equations were solved under the assumption of Newtonian motion (Peters and Mathews 1963; Infeld and MichalskaTrautman 1964), or equations of motion were obtained by inserting approximate solutions of the field equations into the law of motion (2) expanded independently (Peres 1960; Carmeli 1964), which is mathematically inconsistent and may lead to errors of the same order of magnitude as the effects being considered.

The various attempts to carry out the program discussed above differ considerably in details. All methods assume the existence of a global, approximately inertial coordinate system $\left(x^{\alpha}\right)$ in which

$$
g_{\alpha \beta}=\eta_{\alpha \beta}+h_{\alpha \beta}
$$

with

$$
\eta_{\alpha \beta}=\operatorname{diag}(1,-1,-1,-1), \quad\left|h_{\alpha \beta}\right| \ll 1 ;
$$

this is called the "weak field assumption." The major alternative is that between fast and slow approximation methods. In the former ones (4) is chosen such ${ }^{6}$

\footnotetext{
${ }^{5}$ The shortcomings of the derivation are discussed in Havas and Goldberg (1962) and Bonnor (1963).

${ }^{6}$ The choice leading to eq. (9) (the "de Donder condition") is analogous to the choice of the Lorentz gauge in electrodynamics, while the choice leading to instantaneous potentials is analogous
to the Coulomb gauge.
}

that (5) has the form of a quasilinear wave equation

$$
\square_{o} g=T\left(m_{A}, g\right)+\Lambda(g),
$$

which is solved by successive retarded integration to account for (III). In slow motion methods, spacetime is divided into two overlapping zones, a near zone $N$ containing the sources and a far zone $F$ stretching to infinity. In $N$, retardation can be treated as a small correction, and accordingly an approximate solution of (5) is obtained by solving iteratively elliptic equations which can be integrated in terms of instantaneous potentials. The near zone integrations give the potentials ${ }^{N} g$ up to arbitrary harmonic functions only; these functions have to be determined by "matching" the near zone or "inner" expansion of the field to an independently determined far zone or "outer" expansion valid in $F$ only which accounts for the radiation condition (III).

A (perhaps minor) difference between various methods is the use of extended bodies or point particles.

The crucial problem for fast motion methods is to solve equation (9) in sufficient approximation. To achieve that it has been customary to introduce an auxiliary flat metric $\eta$ (supposed to coincide with the "true" metric near past null infinity) as in equation (8a) and to replace (9) by

$$
\square_{\eta} h=T(m, \eta+h)+\Lambda(\eta+h)+M(h),
$$

where $M$ accounts for the "error" caused by the substitution $\square_{g} \rightarrow \square_{\eta}$ on the left-hand side. This has the computational advantage that one can use the flatspace retarded Green's function to solve equation (10) iteratively, starting with $h=0$ on the right-hand side. Clearly, such a reduction (or the corresponding one in the slow motion, near zone equations which leads to the flat space Laplace operator $\Delta$ ) implies the use of the wrong propagators at all steps, and it appears to be unknown whether this leads to serious errors, in the sense that either the iteration does not lead to an approximate solution at all or that it leads to an approximate solution which does not satisfy the boundary condition (III) on $\mathfrak{S}^{-}$, although some information about this question has been obtained by Bird and Dixon (1975) for a simplified model. An alternative method would be to solve (9) in such a way that in the $N$ th step one uses the Green's function associated with the $(N-1)$ th approximation (Thorne and Kovacs 1975).

Unfortunately, the fast motion approach has not been iterated to a high enough order to give conclusive results (for references see Havas 1973). Work on this problem is in progress, however. A variant of the fast approximation (Synge 1970) appears to be free of some of the difficulties mentioned here, but it too has not been carried out far enough to provide an expression for the rate of energy loss due to radiation.

Slow motion methods, on the other hand, have been carried formally to a sufficient order to provide such an expression. ${ }^{7}$ However, a complete matching of the

7 The first consistent calculation [though not fully taking account of condition (III)] was carried out by Goldberg (1955), who 
IN. $N$ and $F$ fields according to the method of singular, matched asymptotic expansions as advocated and 'י applied by Burke $(1969,1971)$ and Burke and Thorne (1970) has not yet been carried out, as far as we know. in: The gaps in the treatments which have been published I'I so far (concerning the results of "matching the post- and post-post-Newtonian near zone solutions outward and then back in," etc.) which have been pointed out by these authors do not appear to have been filled yet. The calculations of Chandrasekhar and Esposito (1970) appear to us to be similarly inconclusive. Although it is emphasized in $\$$ II of their paper that the determination of the lowest-order time-odd terms is preliminary only, an independent proof that these are indeed the lowest order time-odd terms according to the full approximation scheme is not given. Moreover, the $2 \frac{1}{2}$ post-Newtonian equations of motion as well as the metric contain a divergent integral (their eq. [79]) which arises from an integration in $N$ without matching to the appropriate $F$-zone terms. ${ }^{8}$ Although the final formula for radiation loss contains only finite terms, the operations leading to it are not justified, since they are applied to a meaningless, divergent expression.

All of the slow motion methods lead to an expression for the average rate of energy loss due to gravitational radiation of the form

$$
-\frac{d E}{d t}=A \frac{G}{c^{5}} \frac{d^{3} Q_{\alpha \beta}}{d t^{3}} \frac{d^{3} Q^{\alpha \beta}}{d t^{3}}
$$

concluded, however, that the slow motion method was not appropriate for the investigation of gravitational radiation.

\footnotetext{
${ }^{8}$ Anderson and Decanio (1975) are led to an equivalent divergent expression (their eq. [105]) through expansion of retarded potentials in instantaneous ones for the radiation as well as the near zone, in contradiction to their initial assumption that all integrals exist. For the same reason, Epstein and Wagoner (1975) obtain divergent integrals (their eqs. [19], [20], [21], [46b], [47], [48]), contrary to a claim in their paper.
}

where $Q_{\alpha \beta}$ is the quadrupole moment of the system, and $A$ is a numerical constant.

The absence of a term depending on the dipole moment and the particular form (11) of the radiation loss can validly be argued from very general considerations, but not the value of $A$. Exact results for the $F$ zone only imply $A \geq 0$ (Sachs 1962). The most commonly quoted value ${ }^{9}$ (Einstein 1918 ) is $A=1 / 45$; as far as we know, no value greater than this has been claimed. However, all derivations are subject to one or more of the objections discussed earlier, which may invalidate the derivations altogether, or lead to changes of $A$ of the same order of magnitude as the results claimed. Therefore, at present nothing can be said about the value of $A$ (except that it is nonnegative, provided Sachs's assumptions on asymptotic behavior can be justified).

None of the methods attempted so far contains error estimates in a precise mathematical sense; at best, errors have been guessed on the basis of formal "order of magnitude" assignments. Therefore, the predictions concerning period changes of the binary pulsar are not reliable.

In view of this situation it may be advisable to keep an open mind with respect to the question of gravitational radiation damping, and to try other methods or to complete known ones to settle the question, instead of accepting as final results which, however plausible, have been obtained by objectionable methods or by incomplete applications of valid ones. ${ }^{10}$

${ }^{9}$ In comparing the various values of $A$ given in the literature, it should be noted that they depend on the particular definitions of the quadrupole moment adopted by the various authors; the value given here and the form of (11) are those which follow from the convention used by Landau and Lifshitz (1951).

10 Very detailed surveys, from various points of view, of the technical aspects of the problem of radiation loss from isolated gravitating systems will be contained in the Proceedings of the 67th International School of Physics "Enrico Fermi" (held at Varenna, summer 1976) on Isolated Graitating Systems in General Relativity, ed. J. Ehlers (Amsterdam: North-Holland, in press).

\section{APPENDIX}

\section{EXAMPLES ILLUSTRATING EQUATION (3)}

We choose a coordinate system $\left(x^{\alpha}\right)=\left(x^{a}, t\right)\left(\alpha=0,1,2,3 ; a=1,2,3 ; x^{0}=t\right)$ such that the hypersurfaces $t=$ const. are spacelike. The matter variables $m_{A}$ are defined independently of a metric, as required by equation (3).

a) Isentropic perfect fuid. The material is specified by an "equation of state" $p=p(\rho)$. The matter variables $m_{A}(A=$ $1,2,3,4)$ are the energy density $\rho$ and the "coordinate velocity" $v^{a}=d x^{a} / d t$ of the fluid. Then (with $v^{0} \equiv 1$ )

$$
T^{\alpha \beta}=\frac{(\rho+p(\rho)) v^{\alpha} \eta^{\beta}}{g_{\gamma \delta} \nu^{\gamma} v^{\delta}}-p(\rho) g^{\alpha \beta} .
$$

b) Monopole point particle. [This may be considered as a distributional limit of case (a).] The particle is specified by a mass constant $m(>0)$. The matter variables $m_{A}(A=1,2,3)$ are the position coordinates $z^{a}(t)$ of the particle. Then

$$
T^{\alpha \beta}\left(x^{\gamma}\right)=m \int_{-\infty}^{+\infty} \frac{\dot{z}^{\alpha} \dot{z}^{\beta}}{\left(-g g_{\mu \nu} \dot{z}^{\mu} \dot{z}^{\nu}\right)^{1 / 2}} \delta^{4}\left[x^{\gamma}-z^{\gamma}(t)\right] d t,
$$

where $z^{0}=t, \dot{z}^{\alpha}=d z^{\alpha} / d t$, and $\delta$ is the metric-independent Dirac distribution on spacetime, a biscalar density; $g$ now denotes the determinant of $g_{\mu \nu}$. The form $(3 b)$ follow's from equation (2) and the assumption that $T^{\alpha \beta}$ can be represented as an integral over $\delta$ (Tulczyjew 1957; Havas and Goldberg 1962). 


\section{REFERENCES}

Anderson, J. L., and Decanio, T. C. 1975, Gen. Rel. and Grav., 6 197

'I Bird, J., and Dixon, G. 1975, Ann. Phys., 94, 320.

III Bonnor, W. B. 1963, Brit. J. Appl. Phys., 14, 555

Burke, W. L. 1969, California Institute of Technology thesis. 1971, J. Math. Phys., 12, 401

Burke, W. L., and Thorne, K. S. 1970, in Relativity, ed. M. Carmeli, S. J. Fickler, and L. Witten (New York: Plenum Press), p. 209.

Carmeli, M. 1964, Nuovo Cimento 37, 842

Chandrasekhar, S., and Esposito, F. P. 1970, Ap. J., 160, 153.

Chau, W. Y., and Lauterborn, D. 1976, to be published.

D'Eath, P. 1975a, Phys. Rev., D11, 1387. $1975 b$, Caltech preprint OAP-413.

Einstein, A. 1918, Sitzber. Preuss. Akad.Wiss., Physik.-Math. Kl., p. 154.

Epstein, R., and Wagoner, R. V. 1975, Ap. J., 197, 717.

Faulkner, J. 1971, Ap.J. (Letters), 170, L99.

Goldberg, J. N. 1955, Phys. Rev., 99, 1873.

Havas, P. 1973, in Ondes et radiations gravitationelles (Colloques Internationaux CNRS No. 220, Paris), p. 383.

Havas, P., and Goldberg, J. N. 1962, Phys. Rev., 128, 398.

Infeld, L., and Michalska-Trautman, R. 1969, Ann. Phys., 55, 561.

Jürgen Ehlers and Arnold Rosenblum: Max-Planck-Institut für Physik und Astrophysik, Munich, West Germany

Joshua N. Goldberg: Department of Physics, Syracuse University, Syracuse, NY 13210

Peter Havas: Department of Physics, Temple University, Philadelphia, PA 19122
Kalckar, J, and Ulfbeck, O. 1974, K. Danske Vid. Selskab, Mat.fys. Medd., Vol. 39, No. 7.

Landau, L., and Lifshitz, E. 1951, The Classical Theory of Fields (Cambridge: Addison-Wesley), chap 9

Leipold, G. 1976, Institutsbericht MPI-PAE/Astro 75, Munich. Lorentz, H. A. 1909, The Theory of Electrons (Leipzig: B. G. Teubner), pp. 49 and 251.

Newman, E. T., and Unti, T. W. J. 1962, J. Math. Phys., 3, 891.

Ohanian, H. C. 1976, Gravitation and Spacetime (New York: Norton)

Paczynski, B. 1967, Acta Astr., 17, 287

Penrose, R. 1964, in Relativity, Groups and Topology, ed. C. M. de Witt and B. de Witt (New York: Gordon \& Breach), p. 565. Peres, A. 1960, Nuovo Cimento, 15, 351.

Peters, P. C., and Mathews, J. 1963, Phys. Rev., 131, 435.

Pringle, J. E., and Webbink, R. F. 1975, M.N.R.A.S., 172, 493. Sachs, R. K. 1962, Proc. Roy. Soc. (London), 270, 103.

Synge, J. L. 1970, Proc. Roy. Irish Acad., 69, 11.

Thorne, K. S., and Kovacs, S. J. 1975, Ap.J., 200,254

Tulczyjew, W. 1957, Bull. Acad. Polon. Sci. Classe III, 5, 279

Wagoner, R. V. 1975, Ap. J. (Letters), 196, L63.

Weinberg, S. 1972, Gravitation and Cosmology (New Xork: Wiley)

Weyl, H. 1923, Raum, Zeit, Materie (5th ed.; Berlin: SpringerVerlag), $\$ 38$. 